\title{
The Fragmentation of Ethoxylated Surfactants by AP-MALDI-QIT
}

\author{
Scott D. Hanton and David M. Parees \\ Air Products and Chemicals, Inc., Allentown, Pennsylvania, USA
}

\section{Jerry Zweigenbaum}

Agilent Technologies, Inc., Wilmington, Delaware, USA

\begin{abstract}
Matrix-assisted laser desorption/ionization (MALDI) mass spectrometry has become an important technique to characterize the chemical structure of industrial polymer materials. MALDI methods have been developed to address a broad variety of different polymer materials containing different chemistries. One of the key aspects of the typical MALDI experiment is the generation of intact ions. The development of Atmospheric Pressure (AP) MALDI quadrupole ion trap (QIT) instruments has opened another channel to obtaining MS/MS experiments for polymer samples. These experiments provide a new method to obtain chemical structure information from MALDI experiments. Collision-Induced Dissociation (CID) provides an improved MALDI MS/MS experiment that can be done on readily available mass spectrometers. AP MALDI QIT techniques have been successfully applied to a variety of synthetic polymers. This work explores the applicability of AP MALDI QIT methods to relatively low molecular weight ethoxylated surfactants. In these experiments we show the CID fragmentation mass spectra on some ethoxylated surfactants, and demonstrate the existence of analyte matrix clusters. (J Am Soc Mass Spectrom 2006, 17, 453-458) (c) 2006 American Society for Mass Spectrometry
\end{abstract}

$\mathrm{M}$ atrix-assisted laser desorption/ionization (MALDI) mass spectrometry [1-4] has become an important technique to characterize the chemical structure of industrial polymer materials [5-7]. MALDI can generate important data on the telomer repeat units, end groups, and average molecular weights of these materials. MALDI methods have been developed to address a broad variety of different polymer materials containing different chemistries. One of the key aspects of the typical MALDI experiment is the generation of intact ions. MALDI mass spectra generally show little fragmentation. The production of intact ions is vital for the quantitation of average molecular weights, but limits the amount of chemical structural information that can be obtained from the data.

The development of MALDI post-source decay (PSD) techniques has opened a new method to obtain chemical structure information from MALDI experiments [8-10]. Unfortunately, PSD experiments are relatively crude MS/MS experiments. The range of masses submitted for the fragmentation is broad, the mass resolution and mass accuracy of the fragment spectra is not as good as the MALDI data, and there is very

Published online January 25, 2006

Address reprint requests to Dr. S. D. Hanton, Air Products and Chemicals, Inc., 7201 Hamilton Blvd., Allentown, PA 18195, USA. E-mail: HantonSD@ airproducts.com limited control of the energy deposition into the fragmentation process.

The development of atmospheric pressure (AP) MALDI instruments has enabled the utilization of different mass analyzers for MALDI [11, 12]., One of the key benefits to changing the mass analyzer in a MALDI experiment is to significantly improve the MS/MS capability. A quadrupole ion trap (QIT) mass spectrometer is an excellent choice in this regard. Some early work showed examples of high quality MS/MS experiments on MALDI generated peptides [13-16]. The ability to analyze synthetic polymers using AP MALDI methods on a QIT have also been demonstrated [17].

With a QIT mass spectrometer we can accomplish high quality collision-induced dissociation (CID) MS/ MS. The instrument is capable of selecting an individual ion from the mass spectrum for fragmentation. The fragmentation gas and relative energy can be controlled. In addition, higher degrees of fragmentation, $\mathrm{MS}^{\mathrm{n}}$ are possible for intense ions in the mass spectrum.

While we examined a variety of ethoxylated surfactants in this work, this paper will concentrate on results for a commercially available series of telomeric ethoxylated surfactants, Surfynol 4XX (S4XX, XX = 40, 65, and 85). The increasing $X X$ values indicate increasing amounts of ethoxylation on a backbone of 2,4,7,9tetramethyl-5-decyne-4,7-diol (Surfynol 104), as shown in Figure 1.

The performance of these materials as surfactants 


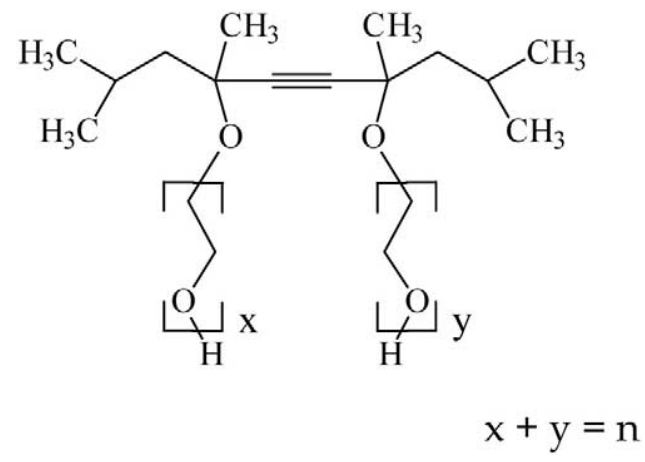

Figure 1. The chemical structure of the S4XX surfactants

depends on the degree of ethoxylation. Surfynols offer several advantages as surfactants: rapid migration, low dynamic surface tension, defoaming, FDA compliance, and stability in high electrolyte brine systems.

Previous gas chromatography (GC) work on S4XX materials shows that for low degree of ethoxylation telomers, the two alcohol sites do not tend to ethoxylate symmetrically [18]. Due to the limitations of volatility, only the low degree of ethoxylation telomers can be studied by GC. In recent vacuum MALDI PSD experiments on S4XX analytes, we attempted to extend the study of these asymmetric telomers [10]. The MALDI PSD experiments produced intense fragments spaced by $44 \mathrm{u}$. The analysis of these fragments showed that they contained ethoxy units from both alcohol sites, and did not produce information regarding the symmetry of the ethoxylation. In addition to the ethoxy-related fragments, we also observed an intense fragment ion that did not have any related ions spaced by $44 \mathrm{u}$ in the higher mass telomers. This fragment ion appeared to have a different fragmentation mechanism than the other observed PSD ions. The assignment and mechanism could not be determined from the PSD data alone. This work was intended to identify the chemical composition of this unknown fragment and determine its fragmentation mechanism.

\section{Experimental}

\section{Chemicals}

The S4XX samples were obtained from Air Products and Chemicals, Inc. (Allentown, PA). The samples were prepared for MALDI using the matrices 2,5-dihydroxybenzoic acid (DHB), $\alpha$-cyano-4-hydroxycinnamic acid (CHCA), and ferulic acid (FA), all obtained from Aldrich. Samples doped with a specific alkali used the $\mathrm{Li}$, $\mathrm{Na}$, or K trifluoroacetic acid (TFA) salt obtained from Aldrich. All chemicals were used as received.

\section{Sample Preparation}

Analytes were prepared as $5 \mathrm{mg} / \mathrm{ml}$ solutions in methanol or tetrahydrofuran (THF). All of the analytes in this study were readily soluble in either solvent. The analyte solutions were mixed $1: 7$ by volume with 0.25 $\mathrm{M}$ matrix solutions in the same solvent. If a sample was doped with a particular alkali cation, an equal amount to the analyte solution of a $35 \mu \mathrm{M}$ M TFA (where $\mathrm{M}=$ $\mathrm{Li}, \mathrm{Na}$, or $\mathrm{K}$ ) solution was added. In most cases, Na was not doped to these samples. If the samples were prepared in soft glass vials, sufficient Na was present in the samples. If Na needed to be excluded from the sample preparation, then the samples were prepared in plastic vials. All samples were deposited by the dry drop method using $\sim 1.0 \mu \mathrm{l}$ of the mixed solution. Samples were allowed to dry under ambient conditions. One 1.0 $\mu \mathrm{l}$ deposition provided sufficient sample for several CID experiments.

\section{Mass Spectrometry}

The AP MALDI experiments were conducted on an Agilent 1100 MSD Trap SL (Wilmington, DE). The AP source was from Agilent Technologies, Inc. The gold substrates were obtained from MassTech (Columbia, $\mathrm{MD})$. The AP source was operated with a drying gas at $50{ }^{\circ} \mathrm{C}$ at a flow rate of $4 \mathrm{~L} / \mathrm{min}$. The nitrogen laser operated at $337 \mathrm{~nm}$. The laser energy was delivered to the target via a fiberoptic cable. Due to the divergence of the laser beam from the end of the fiberoptic cable, higher laser energy was needed for the AP experiments than comparable experiments on a vacuum TOF instrument. Spectra were collected from an average of 16 laser shots from $m / z$ 250-1500. The maximum accumulation time was $2 \mathrm{~s}$.

\section{Results and Discussion}

Vacuum MALDI TOFMS experiments have already characterized the molecular weight distributions, chemical composition, and PSD of S4XX surfactants [10, 18]. AP MALDI can also generate meaningful mass spectra for these materials. Figure 2 (top) shows the AP MALDI mass spectrum of a sample of S465 surfactant with sodium cationization. We clearly see the distribution of ethoxylated telomers. Qualitatively, these results agree well with the previous results. Experiments with different matrices (DHB, CHCA, and FA, data not shown) show that the averaged molecular weights measured in the QIT are not statistically different for the three matrices. The AP MALDI QIT experiments appear to be independent of whether the matrix has been characterized as "hot" or "cold" by TOFMS Experiments19, 20. The middle and bottom mass spectra in Figure 2 show expansions of the spectra obtained with sodium and lithium cationization agents added, respectively. In the bottom spectrum, we also observe a minor series assigned as the matrix adduct of the cationized telomers, $\left[\mathrm{M}+\mathrm{Li}^{+}+\mathrm{LiDHB}\right]^{+}$. These adducts will be discussed more below.

During the acquisition of the mass spectra on the AP MALDI QIT system, we encountered an interesting phenomenon regarding the optimization of the laser 

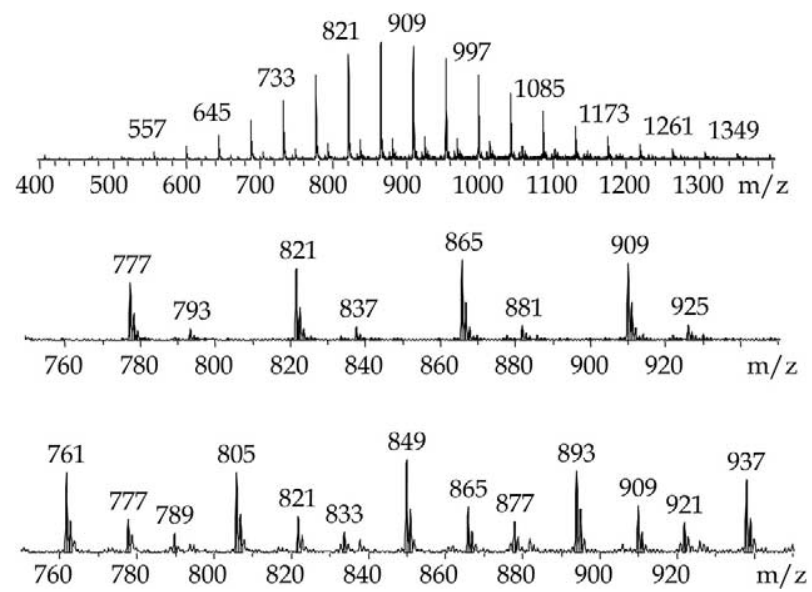

Figure 2. AP MALDI mass spectrum of Surfynol 465. Top: full mass spectrum with $\mathrm{Na}$ cationization agent added. Middle spectrum: expansion of the top spectrum showing $\mathrm{Na}$ (major) and $\mathrm{K}$ (minor) cationization. Bottom spectrum: expansion of a corresponding experiment with Li cationization agent added showing $\mathrm{Li}$ (major) and $\mathrm{Na}, \mathrm{K}$ cationization (minor), and a series assigned as $\mathrm{S} 465+\mathrm{Li}^{+}+\mathrm{DiDHB}$ (minor).

fluence. The AP MALDI source uses a fiberoptic delivery of the laser to the source of the mass spectrometer. Due to the divergence of the laser as it exits the fiberoptic, the spot size at the target is much larger than in a typical vacuum MALDI instrument. The AP MALDI source also uses a heated drying gas that sweeps over the target. Initially, we used a standard drying gas temperature optimized for electrospray ionization of $275^{\circ} \mathrm{C}$. We found that the optimization of the laser was a function of the temperature of the target. When a new target is placed in the instrument we can optimize the laser fluence with respect to the production of the desired MALDI ions. As the target sits in the instrument the heated drying gas impinges the target, heating it up. Over this time the ion intensity observed in the MALDI mass spectra continually decreases. The quality can be restored by reoptimizing the laser fluence. In each case the optimal laser fluence decreases as the target heats up. Since the target has significant mass, the time to reach thermal equilibrium can be significant, extending to a few hours. To ease the process of acquiring data on the instrument, we decreased the drying gas temperature to $50^{\circ} \mathrm{C}$. At this lower temperature, the target comes to thermal equilibrium in just a few minutes. The interplay between the laser fluence optimization and the target temperature may have additional benefits as regards understanding some of the basic mechanisms in MALDI [21].

Figure 3 shows the product ion mass spectrum generated from the $m / z 689\left[\mathrm{M}+\mathrm{Na}^{+}\right]^{+}$ion of the 10 EO telomer. We see the same two fragmentation series observed for PSD of this ion [10]. The more intense series begins at $m / z 479$. This represents the loss of $210 \mathrm{u}$ from the parent ion and is followed by subsequent fragments spaced by $44 \mathrm{u}$. We also see a minor series that begins at $m / z 629$. This represents the loss of $60 \mathrm{u}$

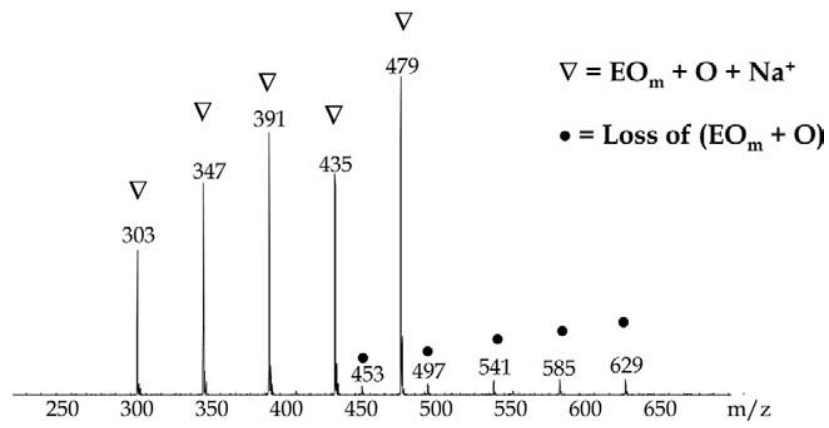

Figure 3. AP MADLI QIT MS/MS of the $m / z 689\left[\mathrm{M}+\mathrm{Na}^{+}\right]^{+}$ion of the $\mathrm{N}=10$ telomer of Surfynol 465 .

from the parent ion and is followed by subsequent fragments spaced by $44 \mathrm{u}$. The main fragmentation series has ions that are spaced by $2 \mathrm{u}$. This is typical of PEG CID, as described in the fundamental work by Selby et al. [22]. We see these major and minor fragment series for all of the S4XX analytes studied. The description of the assignments and mechanisms for these fragment series is described elsewhere, [10] but the $-210 \mathrm{u}$ series has the mass $\mathrm{EO}_{\mathrm{m}}+\mathrm{O}+\mathrm{Na}^{+}$, and the $-60 \mathrm{u}$ series corresponds to the loss of a mass equivalent to $\mathrm{EO}_{\mathrm{m}}+\mathrm{O}$. The primary results of the CID experiment match the primary results of the previous PSD experiments, but the data quality is much higher for the CID experiment. We see much better signal to noise, improved mass resolution on the fragments, and improved mass accuracy of the fragments.

Figure 4 shows the product ion mass spectrum from the $m / z 909\left[\mathrm{M}+\mathrm{Na}^{+}\right]^{+}$ion of the $n=15$ telomer. Again we see the major fragmentation pathway is the loss of $210 \mathrm{u}$ leading to a series of peaks spaced by $44 \mathrm{u}$ and the minor fragmentation pathway is the loss of $60 \mathrm{u}$ followed by peaks spaced by $44 \mathrm{u}$. In addition to the two series observed for the $n=10$ telomer in Figure 3, we see an intense ion at $m / z 733$. This ion does not have any related ions spaced by $44 \mathrm{u}$.

The $m / z 733$ ion corresponds to the loss of $176 \mathrm{u}$. One possible assignment for this loss is $\mathrm{NaDHB}(176 \mathrm{u})$. Since $176 \mathrm{u}$ is the mass of both $\mathrm{EO}_{4}$ and $\mathrm{NaDHB}$, an $n=$ 11 telomer $+\mathrm{NaDHB}+\mathrm{Na}^{+}$also has the nominal mass of $909 \mathrm{u}$. To test this hypothesis, we added $\mathrm{LiOH}$ as a cationization agent. The $\mathrm{Li}^{+}$cationized mass spectrum

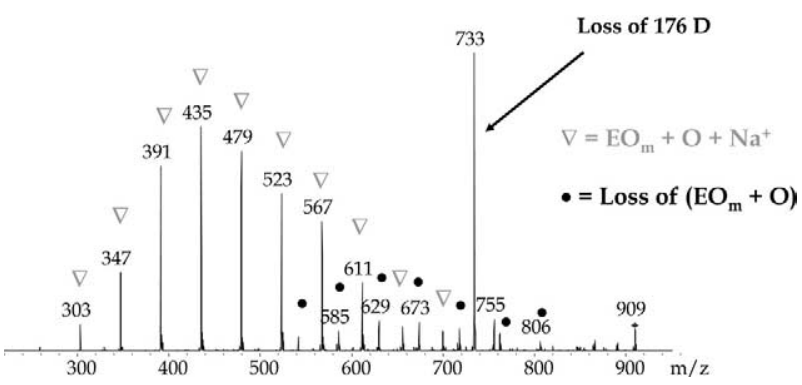

Figure 4. AP MALDI QIT MS/MS of the $m / z 909\left[\mathrm{M}+\mathrm{Na}^{+}\right]^{+}$ion of the $\mathrm{n}=15$ telomer of Surfynol 465 . 


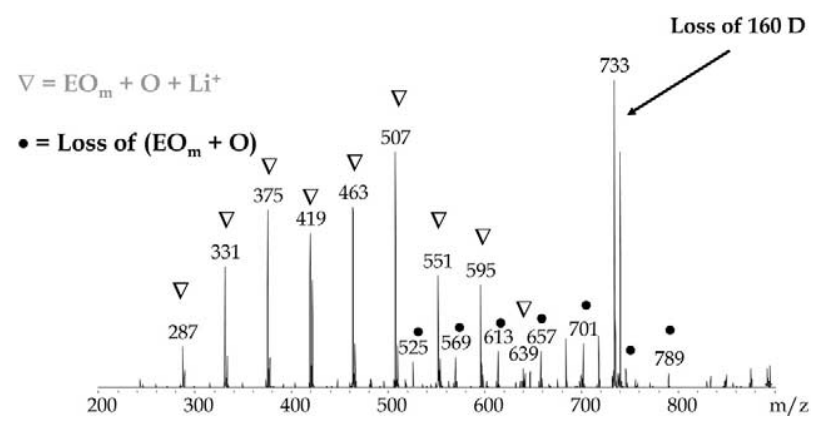

Figure 5. AP MALDI QIT MS/MS of the $m / z 893\left[\mathrm{M}+\mathrm{Li}^{+}\right]^{+}$ion of the $n=15$ telomer of Surfynol 465 .

resulted in intense telomer $+\mathrm{Li}^{+}$ions. The bottom spectrum in Figure 2 shows the presence of matrix adduct ions of the form $\left[\mathrm{M}+\mathrm{Li}^{+}+\mathrm{LiDHB}\right]^{+}$. While adducts with sodium are isobaric with the typical sodium cationized telomers of S465, the lithium cationized/lithium adduct species can be resolved. It should be noted here that we observe very different relative intensities of the lithium adduct ions in different samples. The spectrum shown in Figure 2 contains one of the highest relative intensities of these adduct ions. In other spectra, they are not observed in the MS experiment. We do not yet understand the relative importance of factors such as the matrix/analyte, lithium salt concentration and laser fluence in the production of these ions.

Figure 5 shows the product ion mass spectrum generated from the $m / z 893\left[\mathrm{M}+\mathrm{Li}^{+}\right]^{+}$ion of the $n=15$ telomer. The product ion mass spectrum shows the same series as in Figure 4, except the ions are cationized by $\mathrm{Li}^{+}$instead of $\mathrm{Na}^{+}$. We also see an intense ion at $\mathrm{m} / z$ 733 which corresponds to the loss of $160 \mathrm{u}$. This corresponds to a loss of LiDHB. We can further test our hypothesis by comparing MS [3] mass spectra from the two $\mathrm{m} / \mathrm{z} 733$ ions.

The spectra in Figures 4 and 5 contain another interesting ion. In Figure 4, it is at $m / z$ 755. In Figure 5, it is at $m / z$ 739. In both cases, this is a loss of $154 \mathrm{u}$ from the precursor ion. Apparently, this is a loss of DHB. If so, it would require exchange of sodium for hydrogen on the ethoxylated Surfynol material. These product ions would contain two sodiums. We postulate that this would be the hydrogen on one of the terminal hydroxy groups. If this process is occurring, it is demonstrating something that is not observed in normal MALDI spectra. Sodium/hydrogen exchange leading to ethoxylated Surfynol ions containing two sodium atoms is not observed. Therefore, if this is happening in the MS/MS processes, it may indicate something about both where the NaDHB is bound to the ethoxylated Surfynol molecule, and also imply that the collision energy is sufficient to activate the exchange process. This observation requires additional study.

Figure 6 shows the MS ${ }^{n}$ mass spectra of the $m / z 733$ ions, produced from different routes: MS [2] of the $\mathrm{m} / \mathrm{z}$
$733\left[\mathrm{M}+\mathrm{Na}^{+}\right]^{+}$telomer, MS [3] of the $m / z 909[\mathrm{M}+$ $\left.\mathrm{Na}^{+}\right]^{+}$telomer, and MS [3] of the $m / z 893\left[\mathrm{M}+\mathrm{Li}^{+}\right]^{+}$ telomer ion. All three mass spectra are essentially the same. The $m / z 733$ ions produce the two typical fragment series attributed to S4XX telomers. Interestingly, all of the observed ions are $\mathrm{Na}^{+}$cationized, even the series from the sample that was nominally $\mathrm{Li}^{+}$cationized in the MS spectrum. These data demonstrate that the $\mathrm{Na}^{+}$cation preferentially stays with the S4XX fragments, and the $\mathrm{Li}^{+}$cation preferentially stays with the DHB matrix adduct.

These results confirm our hypothesis of MeDHB (Me $=\mathrm{Na}$ or $\mathrm{Li}$ ) adducts contributing to the observed telomer ion signal. Interestingly, it is $\mathrm{NaDHB}$ which is isobaric with the S4XX telomers. Even in a sample with $\mathrm{LiOH}$ added, which produces a primarily $+\mathrm{Li}^{+}$mass spectrum, we still see evidence for association with an NaDHB adduct.

With the high relative intensity of these matrix adduct fragment ions in the product ion mass spectra, we were concerned about the primary identity of the telomers analyzed in the MS mass spectra. Are the average molecular weights of these materials confounded by matrix adducts? To investigate the relative intensity of these matrix adducts, we looked for the double $\mathrm{Li}$ containing ion $\left(\mathrm{EO}_{\mathrm{m}}+\mathrm{LiDHB}+\mathrm{Li}^{+}\right)$in the mass spectra. We could not identify any ions that can be assigned to this structure. This supports our normal average molecular weight measurements for these materials. It also suggests that the fragmentation to lose a matrix adduct is much more facile than the other fragmentation paths observed for these species.

MS/MS experiments on higher mass telomers yield similar fragments to the lower mass telomers discussed above. For example, the MS [3] product ion spectrum of the $-176 \mathrm{u}$ fragment at $m / z 1262\left[\mathrm{M}+\mathrm{Na}^{+}\right]^{+}$ion of the $n=23$ telomer creates fragments consistent with an S4XX telomer (data not shown). In addition, we see

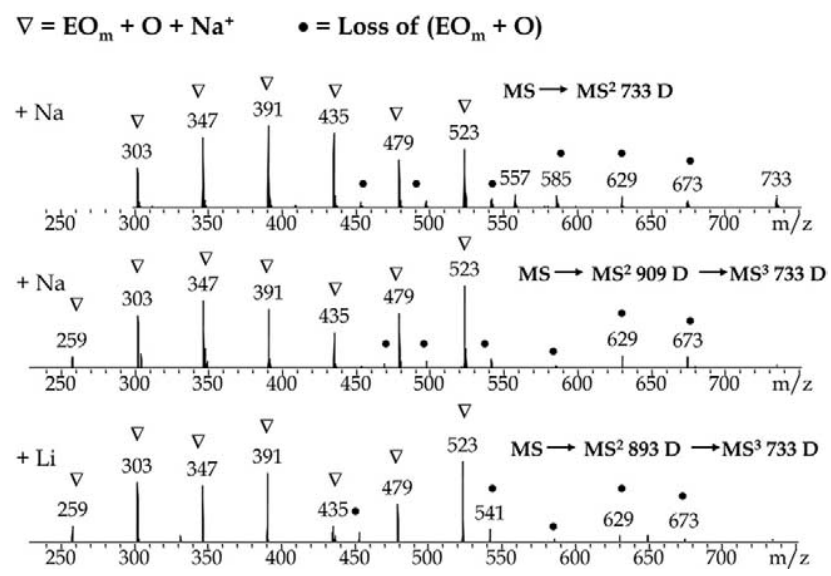

Figure 6. AP MALDI QIT MS ${ }^{n}$ experiments fragmenting the $m / z$ 733 ion of the Surfynol 465 from different routes. Top: MS/MS of the $733\left[\mathrm{M}+\mathrm{Na}^{+}\right]^{+}$ion of the $\mathrm{n}=11$ telomer. Middle: MS/MS 3 of the $909\left[\mathrm{M}+\mathrm{Na}^{+}\right]^{+}$ion of the $\mathrm{n}=15$ telomer. Bottom: $\mathrm{MS} / \mathrm{MS}^{3}$ of the $909\left[\mathrm{M}+\mathrm{Li}^{+}\right]^{+}$ion of the $\mathrm{n}=15$ telomer. 

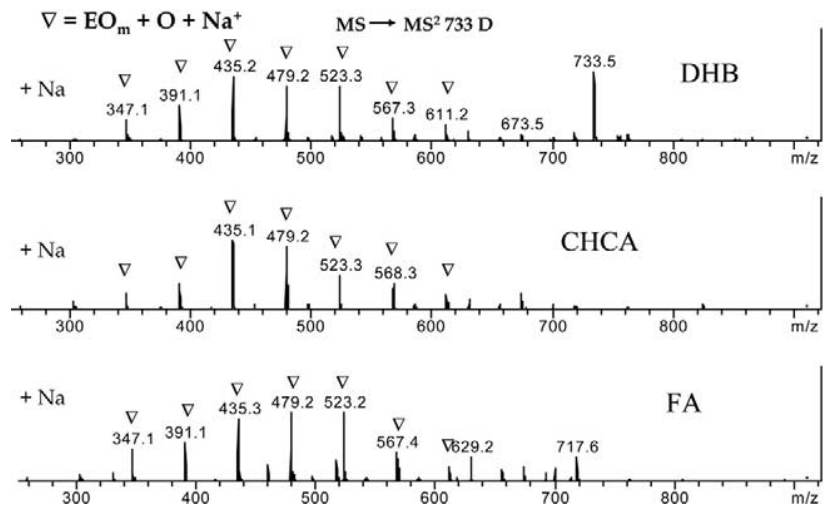

Figure 7. AP MALDI QIT $\mathrm{MS}^{2}$ experiments using different matrices fragmenting the $\mathrm{m} / \mathrm{z} 733$ ion of the Surfynol 465 surfactant. All ions are $\left[\mathrm{M}+\mathrm{Na}^{+}\right]^{+}$of the $\mathrm{n}=11$ telomer. Top: DHB. Middle: CHCA. Bottom: FA. Each matrix produces essentially the same fragments.

another fragment ion at $-176 \mathrm{u}$. Apparently, the $1262 \mathrm{u}$ telomer has contributions from the $n=23$ telomer, the $n=19$ telomer $+\mathrm{NaDHB}$, and the $n=15$ telomer + 2(NaDHB).

To further investigate the nature of the $-176 \mathrm{u}$ ions in these mass spectra, we conducted MS/MS experiments with different matrices. Figure 7 shows the mass spectra of the $m / z 733\left[\mathrm{M}+\mathrm{Na}^{+}\right]^{+}$ion of the $n=11$ telomer obtained using $\mathrm{DHB}, \mathrm{CHCA}$, and FA as the matrix. Each of the matrices produces similar MS/MS fragment ions. Previous studies on TOFMS instruments have described relative differences in how "hot" or "cold" the different MALDI matrices behave in PSD experiments [19]. This effect does not appear to be observed in these QIT experiments.

In these experiments we noted that acquiring MS [3] data on these telomers was difficult. Only the most intense MS/MS ions produced sufficient ion counts to generate any useful MS [3] ion signal. In these telomeric samples the ion intensity is spread over too many different oligomeric species for there to be sufficient intensity in any individual ion to generate MS [3] spectra. If experiments could be run such that significantly increased ion signal were generated, useful $\mathrm{MS}^{\mathrm{n}}$ mass spectra of polymer samples may be accomplished.

One of the objectives of these experiments was to investigate the chemical structure of the $-161 \mathrm{u}$ ions observed in the PSD experiments [10]. Unfortunately, no $-161 \mathrm{u}$ ions were observed in any of the product ion mass spectra. While the $-161 \mathrm{u}$ ions were not observed, we did observe the $-176 \mathrm{u}$ ions, instead. Interestingly, no $-176 \mathrm{u}$ ions were observed by PSD. While we have no data on the $-161 \mathrm{u}$ ions from these experiments, we can speculate about a possibility that would be consistent with both datasets. In a PSD experiment, if the ion fragments promptly, i.e., in the source of the TOFMS, then the velocity would not be correct to be passed by the PSD mass gate. If the ion fragments in a typical metastable process, i.e., in the field-free flight tube, then the PSD mass calibration should be sufficient to assign it correctly. Perhaps the fragment observed at $-161 \mathrm{u}$ actually fragments between the source and the fieldfree flight tube. This would lead to an error in the PSD fragment ion mass assignment. It is possible (although far from proven), that the $-161 \mathrm{u}$ ions observed in the PSD experiment show this type of mass assignment error, and are the same ions observed at $-176 \mathrm{u}$ in the QIT experiments. Much further work would be required to demonstrate this possibility.

\section{Conclusions}

AP MALDI QIT experiments were completed on a series of ethoxylated surfactants. The MS and MS/MS experiments confirmed the previous vacuum MALDI TOFMS and PSD results. These experiments also showed the utility of AP MALDI QIT in the analysis of polymer materials. The MS/MS experiments confirm the power of CID experiments on polymer samples, and the possibility of completing these experiments on relatively inexpensive QIT instruments.

In our MS/MS experiments, we also identified analyte matrix clusters with NaDHB. These clusters are isobaric with the ethoxylated telomers due to the mass overlap between NaDHB and four ethoxy units. The existence of these clusters helps to resolve unassigned peaks from our previous MALDI PSD experiments. We were also successful in obtaining data from some MS [3] experiments, which demonstrated that some of these ions contained multiple matrix cluster species.

\section{Acknowledgments}

The authors thank Air Products and Chemicals, Inc. and Agilent Technologies, Inc. for their support of this research, Professor Kevin Owens from Drexel University and Dr. Andrew Hoteling from Kodak for helpful discussions regarding PSD experiments, and Dr. John Sadowski for critical review of the manuscript.

\section{References}

1. Tanaka, K.; Waki, H.; Ido, Y.; Akita, S.; Yoshido, Y.; Yoshido, T. Protein and Polymer Analyses up to $m / z 100,000$ by Laser Ionization Time-ofFlight Mass Spectrometry. Rapid Commun. Mass Spectrom. 1988, 2, 151.

2. Karas, M.; Hillenkamp, F. Laser Desorption Ionization of Proteins With Molecular Masses Exceeding 10,000 Daltons. Anal. Chem. 1988, 60, 2299.

3. Bahr, U.; Deppe, A.; Karas, M.; Hillenkamp, F.; Giessman, U. Mass Spectrometry of Synthetic Polymers by UV-Matrix-Assisted Laser Desorption/Ionization. Anal. Chem. 1992, 64, 2866.

4. Danis, P.; Karr, D.; Mayer, F.; Holle, A.; Watson, C. The Analysis of Water-Soluble Polymers by Matrix-Assisted Laser Desorption Time-ofFlight Mass Spectrometry. Org. Mass Spectrom. 1992, 27, 843.

5. Hanton, S. D. Mass Spectrometry of Polymers and Polymer Surfaces. Chem. Rev. 2001, 101(2), 527.

6. Nielen, M. W. F. MALDI Time-of-Flight Mass Spectrometry of Synthetic Polymers. Mass Spectrom. Rev. 1999, 18, 309.

7. Montaudo, G.; Lattimer, R, P. Eds.; Mass Spectrometry of Polymers; CRC Press: Boca Raton, FL, 2002.

8. Kaufman, R.; Kirsch, D.; Spengler, B. Int. J. Mass Spectrom. Ion Processes 1997, 165/166, 405.

9. Spengler, B.; Kirsch, D.; Kaufmann, R. Fundamental Aspects of PostSource Decay in Matrix-Assisted Laser Desorption Mass Spectrometry. J. Phys. Chem. 1992, 96, 9678.

10. Hanton, S. D.; Parees, D. M.; Owens, K. G. MALDI-PSD of Low Molecular Weight Ethoxylated Polymers. Int. J. Mass Spectrom. 2004, 238, 257-264.

11. Laiko, V. V.; Moyer, S. C.; Cotter, R. J. Atmospheric Pressure MALDI/ Ion Trap Mass Spectrometry. Anal. Chem. 2000, 72, 5239-5243. 
12. Laiko, V. V.; Baldwin, M. A.; Burlingame, A. L. Atmospheric Pressure Matrix-Assisted Laser Desorption/Ionization Mass Spectrometry. Anal. Chem. 2000, 72, 652-657.

13. Keough, T.; Lacey, M. P.: Strife, R. J. Atmospheric Pressure MatrixAssisted Laser Desorption/Ionization Mass Spectrometry of Sulfonic Acid Derivatized Tryptic Peptides. Rapid Commun. Mass Spectrom. 2001, 15, 2227-2239

14. Moyer, S. C.; Von Seggern, C. E.; Cotter, R. J. Fragmentation of Cationized Phosphotyrosine Containing Peptides by Atmospheric Pressure MALDI/Ion Trap Mass Spectrometry. J. Am. Mass Spectrom. 2003, 14, 581-592.

15. Moyer, S. C.; Cotter, R. J.; Woods, A. S. Fragmentation of phosphopeptides by atmospheric pressure MALDI and ESI/ion trap mass spectrometry. J. Am. Soc. Mass Spectrom. 2002, 13, 274-283.

16. Moyer, S. C.; Marzilli, L. A.; Woods, A. S.; Laiko, V. V.; Doroshenko, V. M. Atmospheric Pressure Matrix-Assisted Laser Desorption/Ionization on a Quadrupole Ion Trap Mass Spectrometer. Int. J. Mass Spectrom. 2003, 226, 133-150.

17. Creaser, C. S.; Reynolds, J. C.; Hoteling, A. J.; Nichols, W. F.; Owens, K. G.Atmospheric Pressure Matrix-Assisted Laser Desorption/Ioniza- tion Ion Trap Mass Spectrometry of Synthetic Polymers: A Comparison with Vacuum Matrix-Assisted Laser Desorption/Ionization Time-ofFlight Mass Spectrometry.Eur. J. Mass Spectrom. 2003, 9, 33-44.

18. Parees, D. M.; Hanton, S. D. Cornelio-Clark, P. A. Willcox, D. A Comparison of Mass Spectrometric Techniques for Generating Molecular Weight Information on a Class of Ethoxylated Oligomers. J. Am. Soc. Mass Spectrom. 1998, 9, 282-291.

19. Karas, M.; Bahr, U.; Strupat, K.; Hillenkamp, F.; Tsarbopoulos, A.; Pramanik, B. N. Matrix Dependence of Metastable Fragmentation of Glycoproteins in MALDI TOF Mass Spectrometry. Anal. Chem. 1995, 67, 675-679.

20. Glückmann, M.; Karas, M. The Initial Velocity and Its Dependence on Matrix, Analyte, and Preparation Method in Ultraviolet Matrix-Assisted Laser Desorption/Ionization. J. Mass Spectrom. 1999, 34, 467-477.

21. Knochenmuss, R. A. Quantitative Model of Ultraviolet Matrix-Assisted Laser Desorption/Ionization Including Analyte Ion Generation. Anal. Chem. 2003, 75, 2199-2207.

22. Selby, T. L.; Wesdemiotis, C.; Lattimer, R. P. Dissociation Characteristics of $[\mathrm{M}+\mathrm{X}]^{+}$ions $(\mathrm{X}=\mathrm{H}, \mathrm{Li}, \mathrm{Na}, \mathrm{K})$ from Linear and Cyclic Polyglycols. J. Am. Soc. Mass Spectrom. 1994, 5, 1081. 